\title{
Accuracy and adequacy of waist circumference cut-off points currently recommended in Brazilian adults
}

\author{
Carolina Avila Vianna ${ }^{1, *}$, Rogério da Silva Linhares ${ }^{1}$, Renata Moraes Bielemann ${ }^{1}$, \\ Eduardo Coelho Machado', David Alejandro González-Chica ${ }^{2}$, \\ Alicia Manitto Matijasevich ${ }^{1}$, Denise Petrucci Gigante ${ }^{1}$ and Iná da Silva dos Santos ${ }^{1}$ \\ 'Post-Graduate Programme in Epidemiology, Federal University of Pelotas, Rua Marechal Deodoro, \\ 1160 - $3^{\circ}$ piso, CEP 96010-220, Pelotas, Rio Grande do Sul, Brazil: ${ }^{2}$ Post-Graduate Programme in Nutrition, \\ Federal University of Santa Catarina, Florianópolis, Santa Catarina, Brazil
}

Submitted 26 June 2012: Final revision received 1 December 2012: Accepted 22 January 2013: First published online 12 March 2013

\begin{abstract}
Objective: To evaluate the adequacy and accuracy of cut-off values currently recommended by the WHO for assessment of cardiovascular risk in southern Brazil.

Design: Population-based study aimed at determining the predictive ability of waist circumference for cardiovascular risk based on the use of previous medical diagnosis for hypertension, diabetes mellitus and/or dyslipidaemia. Descriptive analysis was used for the adequacy of current cut-off values of waist circumference, receiver operating characteristic curves were constructed and the most accurate criteria according to the Youden index and points of optimal sensitivity and specificity were identified.

Setting: Pelotas, southern Brazil.

Subjects: Individuals ( $n$ 2112) aged $\geq 20$ years living in the city were selected by multistage sampling, since these individuals did not report the presence of previous myocardial infarction, angina pectoris or stroke.

Results: The cut-off values currently recommended by WHO were more appropriate in men than women, with overestimation of cardiovascular risk in women. The area under the receiver operating characteristic curve showed moderate predictive ability of waist circumference in men $(0.74,95 \%$ CI $0.71,0.76)$ and women $(0 \cdot 75,95 \%$ CI $0 \cdot 73,0 \cdot 77)$. The method of optimal sensitivity and specificity showed better performance in assessing the accuracy, identifying the values of $95 \mathrm{~cm}$ in men and $87 \mathrm{~cm}$ in women as the best cut-off values of waist circumference to assess cardiovascular risk.

Keywords Conclusions: The cut-off values currently recommended for waist circumference are not suitable for women. Longitudinal studies should be conducted to evaluate the consistency of the findings.

Overweight is considered an important risk factor for morbidity and mortality from CVD. Although there are some ways to diagnose excess body fat, abdominal fat is considered by the WHO as a more accurate predictor of cardiovascular risk, metabolic disorders and death than other anthropometric measures such as $\mathrm{BMI}^{(1,2)}$. The measurement of waist circumference (WC), as an indirect measure of visceral fat, is easy, inexpensive, non-invasive and directly related to BMI. It is also one of the recommended diagnostic procedures to assess abdominal adiposity and risk of CVD, both in clinical practice and in epidemiological studies ${ }^{(3,4)}$.

The WC cut-off points currently recommended to identify the risk of CVD $(80 \mathrm{~cm}$ and $88 \mathrm{~cm}$ for women and $94 \mathrm{~cm}$ and $102 \mathrm{~cm}$ for men, to identify increased and substantially increased risk, respectively) are derived mainly from studies in predominantly European populations ${ }^{(5)}$. Thus, their universal use results in a limiting factor for clinical and epidemiological studies because, as well as sex, age and reproductive history, ethnic characteristics also affect fat distribution, thus influencing body structure and the most appropriate cut-off points which set up the risk of $\mathrm{CVD}^{(6)}$. The need to adopt different cut-off points for different populations was discussed in 2005 by the International Diabetes Federation in its consensus statement for the diagnosis of metabolic syndrome $^{(7)}$. Likewise, in 2008, the WHO published a technical report to reassess the most appropriate WC cutoff points and waist:hip ratios. The report reinforced the recommendation that ethnic or specific differences in 
certain sub-populations must be taken into account to assess these cut-off points in defining the risk of $\mathrm{CVD}^{(6)}$.

Although several studies have evaluated the values of WC which best predict the risk of CVD in different ethnic groups $^{(8,9)}$, the current evidence in South America is insufficient and indicates to keep using the same cut-off points recommended for the European population ${ }^{(9)}$. Only six population-based studies conducted in adults in Brazil which aimed to identify these cut-off values were found. Five were cross-sectional studies ${ }^{(10-14)}$ and only one had a longitudinal design ${ }^{(15)}$. The outcomes considered in these studies were cardiovascular risk factors (hypertension and/or diabetes mellitus), and the values of WC with higher sensitivity and specificity ranged from 85 to $88 \mathrm{~cm}$ in men and from 80 to $86 \mathrm{~cm}$ in women.

The present study assessed the WC cut-off points that best predicted the presence of at least two out of three known risk factors for CVD (hypertension, diabetes mellitus and/or dyslipidaemia, taken as the gold standard for identifying individuals with increased cardiovascular risk) from the use of two different criteria based on analysis of receiver operating characteristic (ROC) curves (Youden index and points of optimal sensitivity and specificity).

\section{Experimental methods}

Between January and June 2010, a cross-sectional population-based study was conducted with adults aged $\geq 20$ years living in the urban area of Pelotas, a city located in southern Brazil with approximately 350000 inhabitants. The study was conducted by the Postgraduate Programme in Epidemiology of the Federal University of Pelotas, as part of a consortium system which included fourteen master's projects, all of which had data collection gathered on a common instrument.

The sample size was set at 2842 individuals, based on the needs of the studies which comprised the research consortium. From this, there was the need to visit 1300 households. The sampling process took place in multiple stages, based on data from the 2000 Census. The 404 census tracts in the city were ordered according to average income of household heads and thereafter 130 sectors were drawn with probability proportional to the number of households. From the identification of the residences of each of the selected tracts, systematic sampling occurred at home. Accordingly, all individuals aged $\geq 20$ years were considered eligible, provided that the following exclusion criteria were not present: inability to stand, limb amputation, and pregnant currently or within the previous 6 months. The interviews were conducted face to face in the household. All interviewers were trained during $40 \mathrm{~h}$. Anthropometric measurements were performed by a team of evaluators trained and standardized in measurement techniques. The acceptable technical errors of measurement were calculated based on the publication of Habicht ${ }^{(16)}$ and the technique for measuring WC followed the recommendations of Lohman et $a l^{(17)}$. The maximum acceptable technical errors of measurement within and between observers, used in the process of standardization for the measurement of WC, were respectively two and three times the error observed by the gold standard. Weight and height were also measured using the same methodology.

WC was measured using a flexible tape (brand Graham Field) with an accuracy of $0 \cdot 1 \mathrm{~cm}$ at the narrowest part of the trunk, identified as the midpoint between the lowest rib margin and the iliac crest. The measurement was taken after expiration. To optimize data quality, we used the average of two measures of WC, obtained at different moments of the interview. Participants were weighed to the nearest $100 \mathrm{~g}$ using a previously calibrated digital scale. Standing height of the barefoot participants was measured to the nearest $1 \mathrm{~mm}$, using aluminium stadiometers of $2 \mathrm{~m}$ length.

In the interview, the participants were also asked about economic, demographic and general health characteristics. Among these data, the ones considered in the present study were gender, skin colour (collected as white, black, brown, yellow or indigenous, subsequently categorized as white or non-white), age, schooling (years of study), current smoking and physical activity during leisure time (from the leisure section of the International Physical Activity Questionnaire $\left.{ }^{(18)}\right)$. Current smokers were participants who smoked at least one cigarette daily over the past $30 \mathrm{~d}$ and participants were considered active if they reached the physical activity guidelines of $150 \mathrm{~min}$ of moderate-to-vigorous physical activity in the last $7 \mathrm{~d}$.

The variables corresponding to the clinical conditions for risk of CVD (hypertension, diabetes mellitus and dyslipidaemia) were collected by the question: 'Has any doctor ever told you that you have...?'. The respondent could answer yes or no for each of the following: '...hypertension or high blood pressure?', '... diabetes mellitus or high sugar?' and '... high cholesterol or triglycerides?'. In order to reduce the likelihood of survival bias in the results and to include only individuals at risk of CVD, information was also collected about previous medical diagnosis of angina pectoris, acute myocardial infarction or stroke through the options '... angina or heart attack?' and '... stroke or cerebral ischaemia?'.

The process of data collection was performed by Personal Digital Assistant (PDA) and the variables (demographic, socio-economic and behavioural) were directly exported to the database. The anthropometric measurements were recorded on paper and were added to the database after a process of double entry and analysis of inconsistencies.

Data analysis was performed using the STATA statistical software package version $12 \cdot 0$. After sample description, mean WC was calculated according to age group (20-39 years, 40-49 years, 50-59 years and $\geq 60$ years) and sex for each of the CVD risk factors considered in the study 
(hypertension, diabetes mellitus and dyslipidaemia) and for the presence of two or more of these factors. The mean WC values obtained were plotted on graphs, stratified by sex and age group, and compared with the cut-off points of WC currently used in the identification of 'increased' and 'substantially increased' risk of CVD ${ }^{(19,20)}$ (respectively, 94 and $102 \mathrm{~cm}$ for men and 80 and $88 \mathrm{~cm}$ for women).

ROC curves were constructed ${ }^{(21,22)}$ to estimate the predictive ability of WC for identifying cardiovascular risk. The Youden index was calculated (highest sensitivity + specificity -1 ) to identify the best cut-off point based on the largest vertical distance between the ROC curve and the diagonal curve, separately for men and women. Cut-off points based on values closer to the point $(0,1)$ or with higher optimal sensitivity and specificity (lowest value $\left.(1-\text { sensitivity })^{2}+(1-\text { specificity })^{2}\right)$ were also calculated. Prevalence, sensitivity, specificity and positive predictive value (PPV) were calculated for WC values closest to the left corner of the curve in relation to CVD risk factors (hypertension, diabetes mellitus and dyslipidaemia). Sensitivity was defined as the proportion of individuals with at least two out of the three risk factors correctly identified by the cut-off values of WC. Specificity was defined as the probability of the point chosen to correctly identify individuals with low risk (none or one risk factor). PPV was defined as the proportion of individuals who, after being identified as high risk by the WC, actually had this high risk (presence of at least two of the three risk factors). The $95 \%$ confidence intervals were calculated for these parameters.

The study was conducted according to the guidelines laid down in the Declaration of Helsinki and all procedures involving human subjects/patients were approved by the Ethics Committee in Research of the Faculty of Medicine at Federal University of Pelotas. Written informed consent was obtained from all participants.

\section{Results}

From a total sample of 3024, we obtained interviews and measured WC for 2454 participants (response rate= $81 \cdot 2 \%)$. The losses were greater in males $(23 \cdot 4 \%)$ than females $(17 \cdot 4 \%)$. The distribution of age, schooling, physical activity and smoking was not different between interviewed and losses or refusals. To avoid survival bias, we included in the analysis only those individuals with risk of developing CVD. Accordingly, we excluded those who reported previous medical diagnoses for angina pectoris, acute myocardial infarction or vascular brain injury $(n=375)$. After exclusions, 2112 individuals participated in our study $(57 \cdot 1 \%$ females $)$.

Table 1 shows the description of the sample, stratified by sex. About $40 \%$ of participants of both sexes were younger than 40 years old and the majority of the sample was made up of white people. More than $70 \%$ of those interviewed had level of schooling up to complete secondary education. As for behavioural characteristics, almost $80 \%$ of females and $69 \%$ of males did not achieve the current guidelines for physical activity during leisure time ( $\geq 150 \mathrm{~min} /$ week). One-quarter of males and $18 \%$ of females were smokers. Over half of the participants were overweight, with more than $20 \%$ being obese. Concerning cardiovascular risk factors, about one-third of the participants reported systemic arterial hypertension (30.4\% of males and $32.5 \%$ of females), while about $8 \%$ were classified with diabetes $(6.3 \%$ of males and $8.4 \%$ of females) and almost $25 \%$ reported dyslipidaemia (17.1\% of males and $24 \cdot 1 \%$ of females). The frequency of participants with at least two out of the three cardiovascular risk factors (hypertension, diabetes and/or dyslipidaemia) was $15 \cdot 8 \%(95 \%$ CI $14 \cdot 2,17 \cdot 3 \%)$ and the prevalence was lower in males $(14 \cdot 1 \%$; $95 \%$ CI $11 \cdot 8,16 \cdot 4 \%)$ than females (17.0\%; 95\% CI $14 \cdot 9,19 \cdot 1 \%)$.

The adequacy of current cut-off points of WC recommended by WHO is shown in Figs 1 and 2. Figure 1 shows the mean WC, according to age and sex, for participants who had one of the three cardiovascular risk factors (hypertension, diabetes or dyslipidaemia) and participants who had none. Participants of both sexes with each risk factor for CVD had greater WC than participants without each risk factor. In males, except those younger than 40 years with hypertension or dyslipidaemia, the cut-off points currently recommended by WHO discriminated individuals with increased risk for CVD. In males without these risk factors, WC increased according to age $(P<0 \cdot 001)$ such that, in general, only in those aged 60 years or more was mean WC close to $94 \mathrm{~cm}$. In males who were older than 40 years and with the cardiovascular risk factors, mean WC was always greater than the cut-off value currently recommended by WHO for increased risk for CVD. However, for females, mean WC was lower than the cut-off point for increased CVD risk only in the youngest group and in those who did not show hypertension, diabetes or dyslipidaemia. Every other group of women had WC greater than $80 \mathrm{~cm}$, including those without hypertension, diabetes or dyslipidaemia. On the other hand, hypertensive females aged 60 years or more and diabetic women who were older than 40 years had mean WC greater than the value for substantially increased risk of CVD $(88 \mathrm{~cm})$. Among females without the risk factors for CVD, a positive association between WC and age $(P<0 \cdot 001)$ was seen in all cases.

Figure 2 compares the mean WC in males and females with at least two or more cardiovascular risk factors and the mean WC in males and females without or with only one risk factor, stratified by age. At all ages, mean WC was greater among those with two or more risk factors for CVD, in both sexes. Among males without or with only one risk factor for CVD, WC increased with age and mean WC was always lower than $94 \mathrm{~cm}$, except in men aged 60 years or more. Nevertheless, males with at least two 
Table 1 Description of the sample according to demographic, socio-economic and behavioural characteristics and prevalence of risk factors for CVD, stratified by sex, Pelotas, southern Brazil, 2010

\begin{tabular}{|c|c|c|c|c|}
\hline \multirow[b]{2}{*}{ Variable } & \multicolumn{2}{|c|}{ Males ( $n$ 906) } & \multicolumn{2}{|c|}{ Females ( $n$ 1206) } \\
\hline & $n$ & $\%$ & $n$ & $\%$ \\
\hline \multicolumn{5}{|l|}{ Age $(n 2112)$} \\
\hline $20-39$ years & 374 & $41 \cdot 3$ & 509 & $42 \cdot 2$ \\
\hline $40-49$ years & 208 & $23 \cdot 0$ & 240 & $19 \cdot 9$ \\
\hline $50-59$ years & 167 & $18 \cdot 4$ & 217 & $18 \cdot 0$ \\
\hline$\geq 60$ years & 157 & $17 \cdot 3$ & 240 & $19 \cdot 9$ \\
\hline \multicolumn{5}{|l|}{ Skin colour/ethnicity ( $n$ 2106) } \\
\hline White & 727 & $80 \cdot 5$ & 1000 & $83 \cdot 1$ \\
\hline Non-white & 176 & $19 \cdot 5$ & 203 & $16 \cdot 9$ \\
\hline \multicolumn{5}{|l|}{ Schooling (completed years) (n 2005) } \\
\hline $0-4$ years & 145 & $16 \cdot 7$ & 206 & $18 \cdot 2$ \\
\hline $5-8$ years & 286 & $32 \cdot 8$ & 318 & $28 \cdot 0$ \\
\hline $9-11$ years & 272 & $31 \cdot 3$ & 319 & $28 \cdot 1$ \\
\hline$\geq 12$ years & 167 & $19 \cdot 2$ & 292 & $25 \cdot 7$ \\
\hline \multicolumn{5}{|l|}{ Economic status* ${ }^{*}(n 2109)$} \\
\hline$A / B$ & 160 & $17 \cdot 7$ & 231 & $19 \cdot 2$ \\
\hline C & 467 & $51 \cdot 7$ & 574 & $47 \cdot 6$ \\
\hline $\mathrm{D} / \mathrm{E}$ & 277 & $30 \cdot 6$ & 400 & $33 \cdot 2$ \\
\hline Physical activity ( $\geq 150 \mathrm{~min} /$ week) ( $n$ 2081) & 277 & $30 \cdot 9$ & 227 & $19 \cdot 2$ \\
\hline Smoking ( $n$ 2112) & 228 & $25 \cdot 2$ & 220 & $18 \cdot 2$ \\
\hline \multicolumn{5}{|l|}{ BMI $\left(\mathrm{kg} / \mathrm{m}^{2}\right)(n 2090)$} \\
\hline$<25 \cdot 0$ & 339 & $37 \cdot 8$ & 491 & $41 \cdot 2$ \\
\hline $25 \cdot 0-29 \cdot 99$ & 371 & $41 \cdot 3$ & 383 & $32 \cdot 1$ \\
\hline$\geq 30 \cdot 0$ & 188 & $20 \cdot 9$ & 318 & $26 \cdot 7$ \\
\hline Hypertension (n 2108) & 274 & $30 \cdot 4$ & 392 & $32 \cdot 5$ \\
\hline Diabetes ( $n$ 2109) & 57 & $6 \cdot 3$ & 101 & $8 \cdot 4$ \\
\hline Dyslipidaemia (n 2104) & 154 & $17 \cdot 1$ & 290 & $24 \cdot 1$ \\
\hline Two or more risk factors $(n 2101) \dagger$ & 127 & $14 \cdot 1$ & 204 & $17 \cdot 0$ \\
\hline
\end{tabular}

${ }^{*}$ Brazilian Association of Research Institute criterion.

tTwo or more risk factors for CVD: hypertension, diabetes and/or dyslipidaemia.

risk factors for CVD had mean WC close to the cut-off value for substantially increased risk for CVD $(102 \mathrm{~cm})$, regardless of age. Concerning females, those with two or more risk factors had mean WC greater than $88 \mathrm{~cm}$, regardless of age. Among females without or with only one risk factor, WC increased according to age and only the youngest women had WC lower than the cut-off value for increased risk for CVD $(80 \mathrm{~cm})$.

Figure 3 shows the ROC curves for males and females. We found that the area under the ROC curve (AUC) showed moderate accuracy of WC to discriminate participants with at least two out of three cardiovascular risk factors studied, in both males $(0 \cdot 74 ; 95 \%$ CI $0 \cdot 71,0 \cdot 76)$ and females $(0 \cdot 75 ; 95 \% \mathrm{CI} 0 \cdot 73,0 \cdot 77)$. The Youden index (defined as the maximum vertical distance between the ROC curve and the diagonal line) ${ }^{(22)}$ showed that the more adequate cut-off values of WC for prediction of at least two risk factors for CVD were $89 \mathrm{~cm}$ in males and $81 \mathrm{~cm}$ in females. On the other hand, using the method of optimal sensitivity and specificity (point on the curve closest to the $(0,1)$ point $\left.{ }^{(22)}\right)$, we found that the most adequate cut-off points for prediction of presence of two or more cardiovascular risk factors for males and females were $95 \mathrm{~cm}$ and $87 \mathrm{~cm}$, respectively.

From WC values closest to the $(0,1)$ point of ROC curves (84 to $104 \mathrm{~cm}$ in males and 78 to $98 \mathrm{~cm}$ in females), we calculated the prevalence of participants with WC greater than or equal to each value, as well as sensitivity, specificity and PPV of these points to predict the presence of at least two cardiovascular risk factors (Table 2). In males, sensitivity and specificity values ranged from $45 \cdot 3 \%$ to $95 \cdot 6 \%$ and from $28 \cdot 7 \%$ to $77 \cdot 4 \%$, respectively. Among females, sensitivity ranged from $36 \cdot 2 \%$ to $92 \cdot 2 \%$, while specificity ranged from $39 \cdot 3 \%$ to $82 \cdot 5 \%$. Considering the optimal sensitivity and specificity criterion, in males the WC value of $95 \mathrm{~cm}$ showed sensitivity of $67 \cdot 2 \%$ and specificity of $60 \cdot 6 \%$. The prevalence at this point was $43 \cdot 2 \%$, and $21 \cdot 1 \%$ were correctly classified with two or more cardiovascular risk factors (PPV). In females, $42.5 \%$ had WC greater than or equal to the cut-off value of $87 \mathrm{~cm}$. This point showed sensitivity of $69.7 \%$, specificity of $62.9 \%$ and PPV of $27.3 \%$. Using the Youden index, the cut-off values of $\geq 89 \mathrm{~cm}$ in males and $\geq 81 \mathrm{~cm}$ in females increased the sensitivity to a little more than $85 \%$, but other validity parameters decreased (specificity lower than $50 \%$, PPV of $19 \cdot 8 \%$ in males and $24 \cdot 7 \%$ in females) and prevalence of abdominal obesity increased (almost $60 \%$ in both sexes).

\section{Discussion}

The present study showed that the currently recommended cut-off points to detect increased risk $(\geq 94 \mathrm{~cm}$ in 

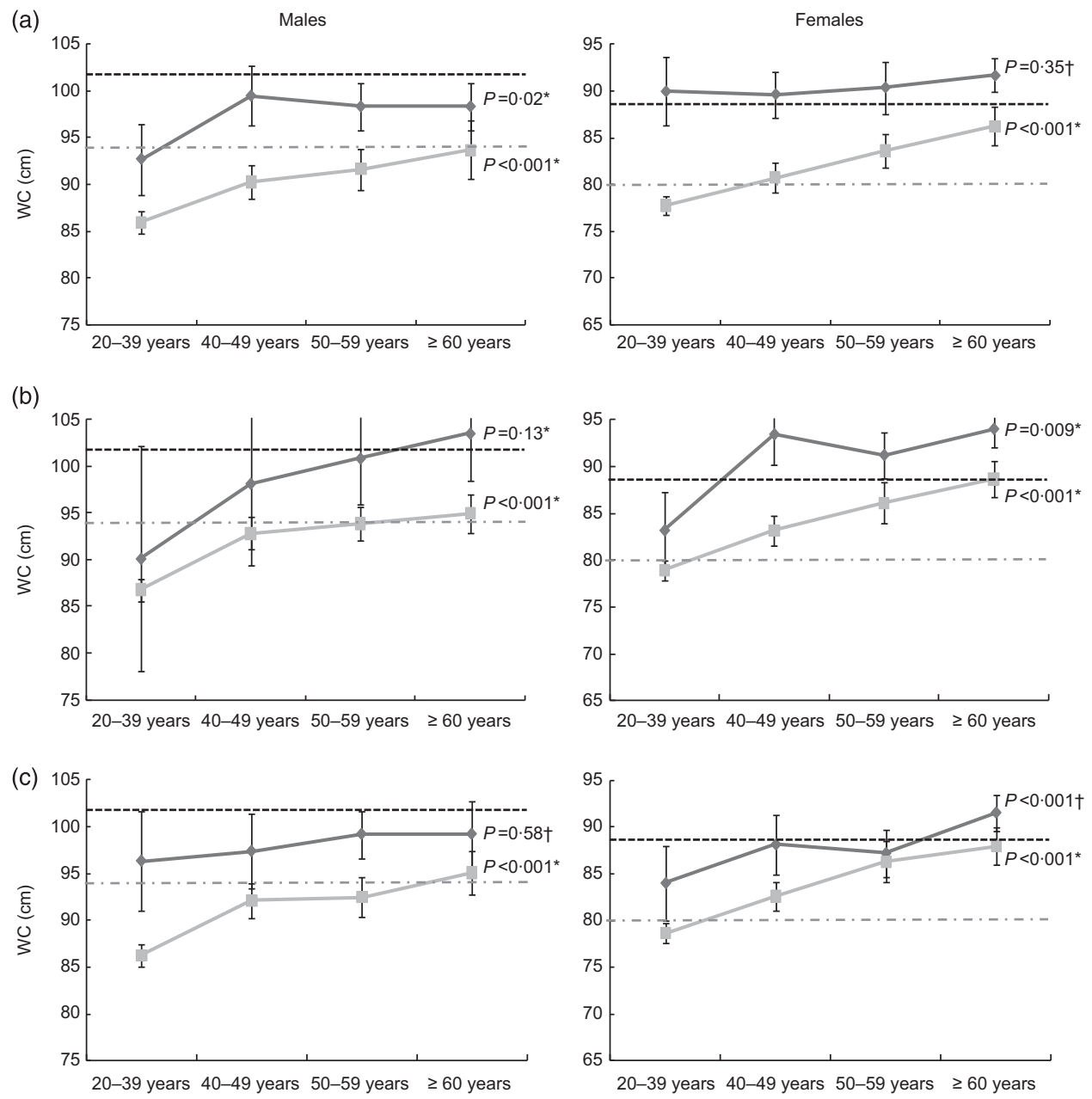

Fig. 1 Waist circumference (WC) according to age among males and females with $(-\bullet)$ or without (- - ) a risk factor for CVD: (a) hypertension, (b) diabetes mellitus and (c) dyslipidaemia; Pelotas, southern Brazil, 2010. Values are means with their standard deviations represented by vertical bars; - . - represents the WHO cut-off for increased risk of CVD $(94 \mathrm{~cm}$ for men, $80 \mathrm{~cm}$ for women); ---. represents the WHO cut-off for substantially increased risk of CVD (102 cm for men, $88 \mathrm{~cm}$ for women). $P$ values for the variation in WC according to age among those with/without risk from *ANOVA test for heterogeneity or $\dagger$ Kruskal-Wallis test

men and $\geq 80 \mathrm{~cm}$ in women) or substantially increased risk ( $\geq 102$ and $\geq 88 \mathrm{~cm}$, respectively) for $\mathrm{CVD}^{(23)}$ seem to have different power of discrimination between sexes. These cut-off points discriminate males at risk better than females at risk, either in the individual assessment of each of the risk factors (hypertension, diabetes mellitus or dyslipidaemia) or when analysed for the presence of at least two of these three factors. Thus, the use of these cut-off points in females leads to a high percentage of misclassification, increasing the number of women diagnosed with abdominal obesity $(60.5 \%$ for values of WC $\geq 80 \mathrm{~cm}$ ) compared with males $(46 \cdot 1 \%$ for values of WC $\geq 94 \mathrm{~cm}$ ). This higher prevalence of abdominal obesity in females is not consistent with the data of morbidity and mortality from CVD, which most often affects males, or with the prevalence of excess body fat assessed by anthropometric indicators such as BMI.

Evaluation of the adequacy of cut-off points for WC recommended by WHO in the Brazilian population is important, since these points were adopted based on individuals of European origin ${ }^{(23)}$. The accumulation of abdominal fat differs between ethnic groups ${ }^{(24)}$, which has led to several studies to assess the most appropriate cut-off points for different populations ${ }^{(9)}$. A greater number of studies have been performed in countries such as China and Japan, with consistent evidence as to the best cut-off points for $\mathrm{WC}$, and new values have been recommended $(80 \mathrm{~cm} \text { in women and } 85 \mathrm{~cm} \text { in men })^{(9)}$. However, in South America, these studies are limited and it is still recommended to maintain the same values as for the European population.

In Brazil, the values found suitable for the prediction of cardiovascular risk from WC ranged from 85 to $88 \mathrm{~cm}$ in males $^{(10-13,15)}$ and from 80 to $86 \mathrm{~cm}$ in females ${ }^{(10-15)}$. These cut-off points were similar to those found in the present study when considering the Youden index $(89 \mathrm{~cm}$ for men and $81 \mathrm{~cm}$ for women), i.e. greater vertical distance between the ROC curve and the diagonal ${ }^{(22)}$, but 
Males

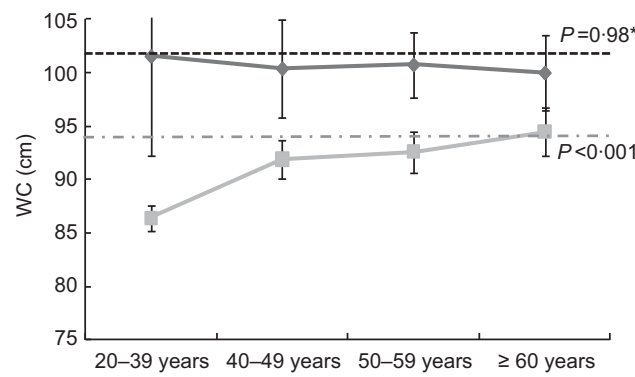

Females

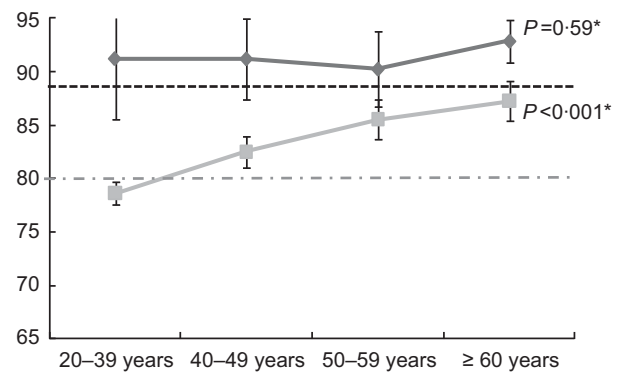

Fig. 2 Waist circumference (WC) according to age among males and females with ( $\geqslant 2$ risk factors, $-\bullet)$ or without $(0$ or 1 risk factor, - -) two or more risk factors for CVD (hypertension, diabetes and/or dyslipidaemia), Pelotas, southern Brazil, 2010. Values are means with their standard deviations represented by vertical bars; - : - : represents the WHO cut-off for increased risk of CVD (94 cm for men, $80 \mathrm{~cm}$ for women); ---- represents the WHO cut-off for substantially increased risk of CVD (102 cm for men, $88 \mathrm{~cm}$ for women). $P$ values for the variation in WC according to age among those with/without risk from *ANOVA test for heterogeneity
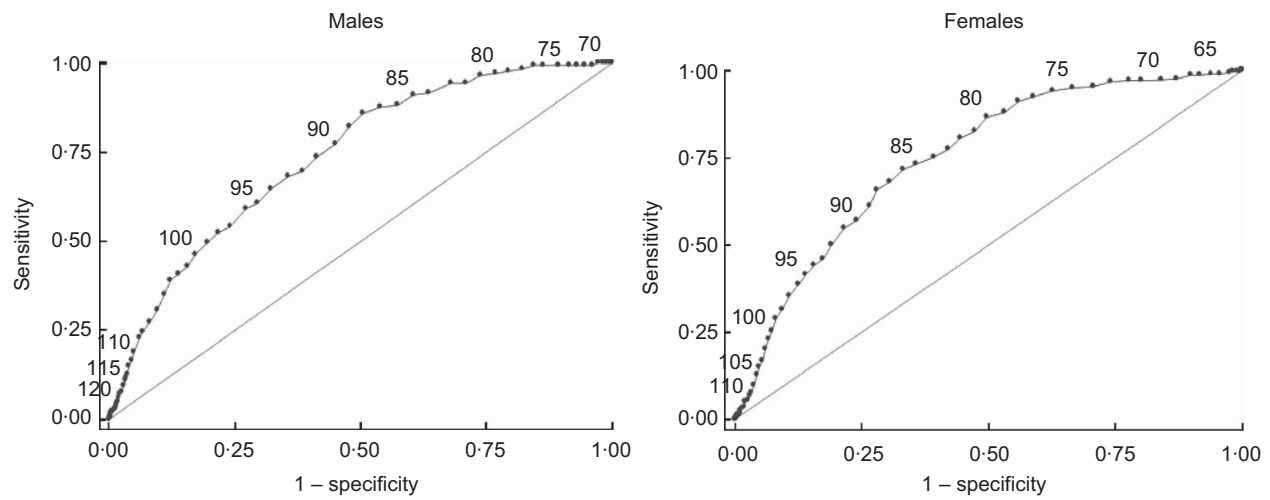

Fig. 3 Receiver operator characteristic $(\mathrm{ROC})$ curves for the predictive value of waist circumference considering two or more cardiovascular risk factors (hypertension, dyslipidaemia and/or diabetes) as the outcome, stratified by sex, Pelotas, southern Brazil, 2010. Area under the ROC curve $(A \cup C)=0.7364$ for males, $A \cup C=0.7492$ for females

lower than those observed with the criterion of optimal sensitivity and specificity values $(95$ and $87 \mathrm{~cm}$, respectively). Among the Brazilian studies, the procedures used to identify these cut-off points were the intersection of the curves of sensitivity and specificity ${ }^{(10-13)}$ or the Youden index ${ }^{(15)}$. The procedures used to define the best cut-off points affect the comparability between studies ${ }^{(6)}$. A systematic review published in 2010 on the values of WC for the prediction of CVD risk ${ }^{(25)}$ showed that, depending on the procedure used, the cut-off points can fluctuate widely in studies conducted in the same country. Despite this limitation, there is no consensus on the best procedure to be used to define the best cut-off points ${ }^{(6)}$.

In the present study, use of the Youden index obtained a high sensitivity and low specificity in predicting the risk of hypertension, diabetes and dyslipidaemia in both males and females. As a result, there was a greater misclassification of individuals with only one risk factor, who ended up being classified as having an increased cardiovascular risk. The use of the optimal sensitivity and specificity criterion may better distinguish between individuals with two or more cardiovascular risk factors and those with up to one factor (highest PPV for both sexes).

As a practical matter, despite the high sensitivity obtained by using the Youden index, its low specificity and high misclassification rate (false positives) limit its use in screening the population, since more than half of the adult population would be identified as having increased risk for developing CVD. Consequently, there is a greater impact on health services, as well as higher costs arising subsequently in the diagnostic evaluation of individuals at low risk with positive screening. This could be visually assessed by applying the cut-off points obtained from both procedures to Figs 1 and 2 in this paper. Using the values of optimal sensitivity and specificity in males $(95 \mathrm{~cm})$ would maintain the ability to discriminate between individuals with and without hypertension, diabetes mellitus and/or dyslipidaemia at different ages. In females, with the cut-off point of $87 \mathrm{~cm}$ it would be possible to identify more clearly who are the people having or not having these risk factors (individual or combined factors). 
Table 2 Prevalence, sensitivity (ST), specificity (SP) and positive predictive value (PPV) of cut-off points of waist circumference (WC) for prediction of two or more cardiovascular risk factors (hypertension, diabetes and/or dyslipidaemia) in males and females from Pelotas, southern Brazil, in 2010

\begin{tabular}{|c|c|c|c|c|c|c|c|c|c|c|c|}
\hline \multirow{3}{*}{$\begin{array}{l}\text { WC cut-off } \\
\text { point }(\mathrm{cm})\end{array}$} & \multicolumn{5}{|c|}{ Males } & \multicolumn{6}{|c|}{ Females } \\
\hline & \multicolumn{2}{|c|}{ Prevalence } & \multirow[b]{2}{*}{ ST (\%) } & \multirow[b]{2}{*}{ SP (\%) } & \multirow[b]{2}{*}{ PPV (\%) } & \multirow{2}{*}{$\begin{array}{l}\text { WC cut-off } \\
\text { point }(\mathrm{cm})\end{array}$} & \multicolumn{2}{|c|}{ Prevalence } & \multirow[b]{2}{*}{ ST (\%) } & \multirow[b]{2}{*}{ SP (\%) } & \multirow[b]{2}{*}{ PPV (\%) } \\
\hline & $n$ & $\%$ & & & & & $n$ & $\%$ & & & \\
\hline$\geq 84$ & 755 & $74 \cdot 6$ & $95 \cdot 6$ & $28 \cdot 7$ & $17 \cdot 4$ & $\geq 78$ & 862 & $66 \cdot 0$ & $92 \cdot 2$ & $39 \cdot 3$ & $23 \cdot 3$ \\
\hline$\geq 85$ & 716 & $70 \cdot 8$ & $92 \cdot 7$ & $32 \cdot 7$ & $17 \cdot 7$ & $\geq 79$ & 829 & $63 \cdot 4$ & $90 \cdot 4$ & $42 \cdot 0$ & $23 \cdot 8$ \\
\hline$\geq 86$ & 690 & $68 \cdot 2$ & $92 \cdot 0$ & 35.5 & $18 \cdot 3$ & $\geq 80$ & 791 & 60.5 & $85 \cdot 8$ & $44 \cdot 5$ & $23 \cdot 6$ \\
\hline$\geq 87$ & 663 & $65 \cdot \overline{5}$ & $89 \cdot 8$ & $38 \cdot 3$ & $18 \cdot 6$ & $\geq 81$ & 753 & $57 \cdot 6$ & $85 \cdot 3$ & $47 \cdot 9$ & $24 \cdot 7$ \\
\hline$\geq 88$ & 635 & $62 \cdot 7$ & $89 \cdot 1$ & $41 \cdot 4$ & $19 \cdot 2$ & $\geq 82$ & 721 & $55 \cdot 2$ & $81 \cdot 7$ & $50 \cdot 1$ & $24 \cdot 7$ \\
\hline$\geq 89$ & 606 & $59 \cdot 9$ & $87 \cdot 6$ & 44.5 & $19 \cdot 8$ & $\geq 83$ & 690 & $52 \cdot 8$ & $79 \cdot 8$ & $52 \cdot 6$ & $25 \cdot 2$ \\
\hline$\geq 90$ & 578 & $57 \cdot 1$ & $82 \cdot 5$ & $46 \cdot 9$ & $19 \cdot 6$ & $\geq 84$ & 660 & 50.5 & $76 \cdot 6$ & $54 \cdot 7$ & $25 \cdot 3$ \\
\hline$\geq 91$ & 551 & $54 \cdot 4$ & $78 \cdot 1$ & $49 \cdot 3$ & $19 \cdot 4$ & $\geq 85$ & 625 & $47 \cdot 8$ & $73 \cdot 4$ & $57 \cdot 3$ & $25 \cdot 6$ \\
\hline$\geq 92$ & 519 & $51 \cdot 3$ & 74.5 & $52 \cdot 3$ & $19 \cdot 7$ & $\geq 86$ & 587 & 44.9 & $71 \cdot 6$ & $60 \cdot 4$ & $26 \cdot 6$ \\
\hline$\geq 93$ & 493 & $48 \cdot 7$ & $71 \cdot 5$ & 54.9 & $19 \cdot 9$ & $\geq 87$ & 556 & $42 \cdot 5$ & $69 \cdot 7$ & $62 \cdot 9$ & $27 \cdot 3$ \\
\hline$\geq 94$ & 467 & $46 \cdot 1$ & $70 \cdot 8$ & $57 \cdot 7$ & $20 \cdot 8$ & $\geq 88$ & 523 & $40 \cdot 0$ & $66 \cdot 5$ & $65 \cdot 3$ & $27 \cdot 7$ \\
\hline$\geq 95$ & 437 & $43 \cdot 2$ & $67 \cdot 2$ & $60 \cdot 6$ & $21 \cdot 1$ & $\geq 89$ & 491 & $37 \cdot 6$ & $63 \cdot 8$ & $67 \cdot 7$ & $28 \cdot 3$ \\
\hline$\geq 96$ & 412 & $40 \cdot 7$ & $62 \cdot 8$ & $62 \cdot 7$ & $20 \cdot 9$ & $\geq 90$ & 466 & $35 \cdot 7$ & $59 \cdot 6$ & $69 \cdot 1$ & $27 \cdot 9$ \\
\hline$\geq 97$ & 392 & $38 \cdot 7$ & $61 \cdot 3$ & $64 \cdot 8$ & $21 \cdot 4$ & $\geq 91$ & 434 & $33 \cdot 2$ & $56 \cdot 0$ & $71 \cdot 3$ & $28 \cdot 1$ \\
\hline$\geq 98$ & 363 & 35.9 & $56 \cdot 9$ & $67 \cdot 4$ & $21 \cdot 5$ & $\geq 92$ & 408 & $31 \cdot 2$ & $53 \cdot 7$ & $73 \cdot 3$ & $28 \cdot 7$ \\
\hline$\geq 99$ & 345 & $34 \cdot 1$ & $56 \cdot 2$ & $69 \cdot 4$ & $22 \cdot 3$ & $\geq 93$ & 375 & $28 \cdot 7$ & $49 \cdot 1$ & $75 \cdot 4$ & 28.5 \\
\hline$\geq 100$ & 326 & $32 \cdot 2$ & $54 \cdot 0$ & $71 \cdot 2$ & $22 \cdot 7$ & $\geq 94$ & 351 & $26 \cdot 9$ & $44 \cdot 5$ & $76 \cdot 7$ & $27 \cdot 6$ \\
\hline$\geq 101$ & 306 & $30 \cdot 2$ & $51 \cdot 8$ & $73 \cdot 1$ & $23 \cdot 2$ & $\geq 95$ & 328 & $25 \cdot 1$ & $48 \cdot 2$ & $78 \cdot 3$ & $28 \cdot 0$ \\
\hline$\geq 102$ & 290 & $28 \cdot 7$ & $48 \cdot 2$ & $74 \cdot 4$ & $22 \cdot 8$ & $\geq 96$ & 309 & $23 \cdot 6$ & 39.9 & $79 \cdot 6$ & $28 \cdot 2$ \\
\hline$\geq 103$ & 275 & $27 \cdot 2$ & $46 \cdot 7$ & $75 \cdot 9$ & $23 \cdot 3$ & $\geq 97$ & 291 & $22 \cdot 3$ & $37 \cdot 6$ & $80 \cdot 8$ & $28 \cdot 2$ \\
\hline$\geq 104$ & 260 & $25 \cdot 7$ & $45 \cdot 3$ & $77 \cdot 4$ & $23 \cdot 8$ & $\geq 98$ & 270 & $20 \cdot 7$ & $36 \cdot 2$ & 82.5 & $29 \cdot 3$ \\
\hline
\end{tabular}

Another aspect to be considered in comparison with other studies is the different end points used. In 2005, Pitanga and Lessa conducted a cross-sectional study in north-eastern Brazil that compared various anthropometric indicators, including WC, to identify which one best discriminated high coronary risk (determined based on an index that included age, blood pressure, total cholesterol, HDL-cholesterol, smoking and diagnosis of diabetes mellitus) ${ }^{(11)}$. In the same sample, other authors identified the best cut-off points of WC for the diagnosis of diabetes mellitus and obesity ${ }^{(12)}$. Two other studies used just hypertension as the outcome, being a longitudinal type study performed in southern Brazil ${ }^{(15)}$ and a cross-sectional study in the mid-west ${ }^{(10)}$. In 2006, Ferreira et al. used only dyslipidaemia as outcome ${ }^{(13)}$. Among those who have used various criteria to define the risk of $\mathrm{CVD}^{(11,12,26)}$, the cut-off point of WC among females was higher than that provided by studies using a single health condition $^{(10,13,15)}$. The first three studies agreed on the procedure used to measure WC based on the narrowest point of the trunk, rather than the midpoint between the costal margin and the iliac crest - criteria used by other Brazilian papers - which can also influence the results ${ }^{(6)}$. It is also important to note that only four studies used population-based samples ${ }^{(10-12,15)}$, which can also affect the comparability of results.

Regarding the predictive ability of WC for cardiovascular risk assessment, the AUC observed was 0.73 (95\% CI 0.71, $0 \cdot 76$ ) for males and 0.74 (95\% CI $0.73,0 \cdot 77)$ for females in the present study. The prediction of cardiovascular risk from WC has been considered more applicable than from other anthropometric indices such as $\mathrm{BMI}^{(27)}$, the main limitation of this last indicator being its inability to distinguish between muscle and adipose tissue ${ }^{(28)}$. The AUC in that study ${ }^{(27)}$ was higher for WC than for BMI, in males (AUC for BMI $=0 \cdot 69$; $95 \%$ CI $0 \cdot 65,0 \cdot 74$ ) and females (AUC for BMI $=0 \cdot 70 ; 95 \%$ CI $0.67,0 \cdot 74 ; P$ value of the difference with AUC for WC $<0.001$ in both cases), suggesting that the former was a better test to evaluate cardiovascular risk. The AUC values for WC that we found were also similar to those reported in a meta-analysis published recently ${ }^{(27)}$. However, the meta-analysis evaluated the predictive ability of WC for each cardiovascular risk factor, whereas the data presented here for the ROC curves refer to the presence of two or more cardiovascular risk factors. Nevertheless, results from prospective studies suggest that BMI has similar ability to predict CVD risk factors as measurements of central adiposity. The Caerphilly Prospective Study, a British cohort of men, showed no differences in the strengths of association with incident diabetes between BMI, WC and two other indicators of abdominal obesity ${ }^{(29)}$. On the other hand, the British Women's Heart and Health Study ${ }^{(30)}$ found that WC and waist:height ratio were more strongly associated with diabetes than BMI. New prospective studies are necessary to elucidate these differences.

One possible limitation of our study was the use of self-reported data for the diagnosis of hypertension, diabetes and dyslipidaemia. The choice of these diseases was based on their relevance in the prediction of cardiovascular risk and they are recommended in studies to this end by the $\mathrm{WHO}^{(6)}$. Although this is self-reported information, a previous study in Pelotas showed that 
self-report to identify the prevalence of hypertension had a sensitivity of $84.3 \%$, specificity of $87 \cdot 5 \%$ and reliability of $80.0 \%{ }^{(31)}$. Another study in Minas Gerais also assessed the self-report of hypertension in older adults and found a sensitivity of $72 \cdot 1 \%$ and specificity of $86 \cdot 4 \%{ }^{(32)}$. In relation to diabetes, Dode and Santos studied the validity of self-reported gestational diabetes in 2009, and found a relevant sensitivity $(72.9 \%)$ and high specificity (99.0\%) and accuracy $(97 \cdot 9 \%)^{(33)}$. Even with higher accuracy, a possible bias due to self-reported compared with measured outcomes would be a higher misclassification of the disease in young compared with older age groups ${ }^{(31,32)}$. This possible bias could explain the greater accuracy of WC at older ages.

There were losses of almost $20 \%$ of individuals eligible for the study, which were higher among males. Even if these losses are considered a limitation of the study, they only influence the accuracy of the results, resulting in no bias, since the main findings consist of descriptive and predictive analysis of the cut-off points of WC. Another important issue to consider is the study design. Longitudinal studies are better for assessment of disease risks than cross-sectional ones that cannot distinguish between risk factors and prognostic factors. However, the exclusion of individuals with pre-existing CVD is a strong point of the study, which could affect the results to represent the surviving cases, possibly related to good prognosis. Other strengths were the two methodological steps to reduce errors in the measurements of $\mathrm{WC}$, as well as the use of two different criteria for assessing the best cut-off points. Finally, we have to consider the external validity of our results. Brazil is a country with more than 190 million inhabitants and, according to the Brazilian census of 2010 , skin colour was self-classified as white for $47 \cdot 7 \%$ and black for $7 \cdot 6 \%$ of the citizens ${ }^{(34)}$. In Pelotas, most of the inhabitants self-reported their skin colour as white $(80 \cdot 3 \%)$, but the proportion of blacks $(10 \cdot 7 \%)$ is higher than in other cities in southern Brazil. These demographic differences should be considered when comparing our results, as previous studies have shown that body composition and cut-offs depend on race/ethnicity ${ }^{(6,9,24)}$.

\section{Conclusions}

The results of our study suggest that the cut-off values of WC currently recommended for screening for CVD risk are more appropriate for males than females, incurring an overestimation of the risk of CVD among females, due to low cut-off values of this population. This error causes the positive screening of women without a real high risk, which in terms of public health would lead to greater demand for consultations and diagnostic tests, resulting in overloading existing professionals and higher spending on health. Thus, it is important that further studies be conducted in other regions of Brazil, considering not only the different ethnic origins that Brazil presents, but also the methodology used, thus helping to identify the cut-off points of WC best suited for the prediction of CVD risk.

\section{Acknowledgements}

Sources of funding: The authors wish to acknowledge the Brazilian agency Coordenação de Aperfeiçoamento de Pessoal de Nivel Superior (CAPES) for funding and scholarship support to R.M.B. Conflicts of interest: The authors report no conflict of interest. Authors' contributions: C.A.V., R.S.L., R.M.B. and E.C.M. conceptualized the study, coordinated the field work, ran the analyses and wrote the manuscript. D.P.G., A.M.M. and I.S.S. contributed to the writing and revision of the manuscript. D.A.G.-C. conceptualized the study, ran the analyses and contributed to the writing and revision of the manuscript.

\section{References}

1. World Health Organization (1998) Obesity: Preventing and Managing the Global Epidemic. Report of a WHO Consultation on Obesity, Geneva, 3-5 June 1997. Geneva: WHO.

2. Huxley R, Mendis S, Zheleznyakov E et al. (2010) Body mass index, waist circumference and waist:hip ratio as predictors of cardiovascular risk - a review of the literature. Eur J Clin Nutr 64, 16-22.

3. Pouliot MC, Despres JP, Lemieux S et al. (1994) Waist circumference and abdominal sagittal diameter: best simple anthropometric indexes of abdominal visceral adipose tissue accumulation and related cardiovascular risk in men and women. Am J Cardiol 73, 460-468.

4. Oka R, Miura K, Sakurai M et al. (2009) Comparison of waist circumference with body mass index for predicting abdominal adipose tissue. Diabetes Res Clin Pract 83, 100-105.

5. Han TS, van Leer EM, Seidell JC et al. (1995) Waist circumference action levels in the identification of cardiovascular risk factors: prevalence study in a random sample. BMJ 311, 1401-1405.

6. World Health Organization (2008) Waist Circumference and Waist-Hip Ratio: Report of a WHO Expert Consultation. Geneva: WHO.

7. Alberti KG, Zimmet P \& Shaw J (2006) Metabolic syndrome a new world-wide definition. A Consensus Statement from the International Diabetes Federation. Diabet Med 23, 469-480.

8. Herrera VM, Casas JP, Miranda JJ et al. (2009) Interethnic differences in the accuracy of anthropometric indicators of obesity in screening for high risk of coronary heart disease. Int J Obes (Lond) 33, 568-756.

9. Lear SA, James PT, Ko GT et al. (2010) Appropriateness of waist circumference and waist-to-hip ratio cutoffs for different ethnic groups. Eur J Clin Nutr 64, 42-61.

10. Peixoto Mdo R, Benicio MH, Latorre Mdo R et al. (2006) Waist circumference and body mass index as predictors of hypertension. Arq Bras Cardiol 87, 462-470.

11. Pitanga FJ \& Lessa I (2005) Anthropometric indexes of obesity as an instrument of screening for high coronary risk in adults in the city of Salvador - Bahia. Arq Bras Cardiol 85, 26-31.

12. Barbosa PJ, Lessa I, de Almeida Filho N et al. (2006) Criteria for central obesity in a Brazilian population: impact on metabolic syndrome. Arq Bras Cardiol 87, 407-414. 
13. Ferreira MG, Valente JG, Goncalves-Silva RM et al. (2006) Accuracy of waist circumference and waist-to-hip ratio as predictors of dyslipidemia in a cross-sectional study among blood donors in Cuiaba, Mato Grosso State, Brazil. Cad Saude Publica 22, 307-314.

14. Velásquez-Meléndez G, Kac G, Valente JG et al. (2002) Evaluation of waist circumference to predict general obesity and arterial hypertension in women in Greater Metropolitan Belo Horizonte, Brazil. Cad Saude Publica 18, $765-771$.

15. Gus M, Cichelero FT, Moreira CM et al. (2009) Waist circumference cut-off values to predict the incidence of hypertension: an estimation from a Brazilian populationbased cohort. Nutr Metab Cardiovasc Dis 19, 15-19.

16. Habicht JP (1974) Standardization of quantitative epidemiological methods in the field. Bol Oficina Sanit Panam 76, 375-384.

17. Lohman TG, Roche AF, Martorell R et al. (1988) Anthropometric Standardization Reference Manual. Champaign IL: Human Kinetics Books.

18. Craig CL, Marshall AL, Sjostrom M et al. (2003) International physical activity questionnaire: 12-country reliability and validity. Med Sci Sports Exerc 35, 1381-1395.

19. US National Institutes of Health (1998) Clinical Guidelines on the Identification, Evaluation, and Treatment of Overweight and Obesity in Adults - The Evidence Report. Obes Res 6, Suppl. 2, S51-S209.

20. World Health Organization (2000) Obesity: Preventing and Managing the Global Epidemic. Report of a WHO Consultation. WHO Technical Report Series no. 894. Geneva: WHO

21. Zweig MH \& Campbell G (1993) Receiver-operating characteristic (ROC) plots: a fundamental evaluation tool in clinical medicine. Clin Chem 39, 561-577.

22. Akobeng AK (2007) Understanding diagnostic tests 3: receiver operating characteristic curves. Acta Paediatr 96, 644-647.

23. Lean ME, Han TS \& Morrison CE (1995) Waist circumference as a measure for indicating need for weight management. BMJ 311, 158-161.
24. Lear SA, Humphries KH, Kohli S et al. (2007) Visceral adipose tissue accumulation differs according to ethnic background: results of the Multicultural Community Health Assessment Trial (M-CHAT). Am J Clin Nutr 86, 353-359.

25. Wang Z, Ma J \& Si D (2010) Optimal cut-off values and population means of waist circumference in different populations. Nutr Res Rev 23, 191-199.

26. Almeida RT, Almeida MM \& Araujo TM (2009) Abdominal obesity and cardiovascular risk: performance of anthropometric indexes in women. Arq Bras Cardiol 925, 375-380.

27. Lee CM, Huxley RR, Wildman RP et al. (2008) Indices of abdominal obesity are better discriminators of cardiovascular risk factors than BMI: a meta-analysis. $J$ Clin Epidemiol 61, 646-653.

28. Yajnik CS \& Yudkin JS (2004) The Y-Y paradox. Lancet $363,163$.

29. Taylor AE, Ebrahim S, Ben-Shlomo Y et al. (2010) Comparison of the associations of body mass index and measures of central adiposity and fat mass with coronary heart disease, diabetes, and all-cause mortality: a study using data from 4 UK cohorts. Am J Clin Nutr 91, 547-556.

30. Lawlor DA, Ebrahim S \& Davey Smith G (2002) The association between components of adult height and type II diabetes and insulin resistance: British Women's Heart and Health Study. Diabetologia 45, 1097-1106.

31. Chrestani MA, Santos Ida S \& Matijasevich AM (2009) Self-reported hypertension: validation in a representative cross-sectional survey. Cad Saude Publica 25, 2395-2406.

32. Lima-Costa MF, Peixoto SV \& Firmo JO (2004) Validity of self-reported hypertension and its determinants (the Bambui study). Rev Saude Publica 38, 637-642.

33. Dode MA \& Santos IS (2009) Validity of self-reported gestational diabetes mellitus in the immediate postpartum. Cad Saude Publica 25, 251-258.

34. Brazilian Institute of Geography and Statistics (2011) 2010 Population Census. http://www.ibge.gov.br/home/estatistica/ populacao/censo2010/default.shtm (accessed March 2012). 